\title{
Host plants of Cerambycidae and Vesperidae (Coleoptera, Chrysomeloidea) from South America
}

\author{
Vanessa S. Machado ${ }^{1}$, Juan P. Botero ${ }^{1}$, Allan Carelli ${ }^{1}$, Mario Cupello ${ }^{1}$, Hingrid Y. Quintino ${ }^{1} \&$ Marianna V. P. Simões $^{1}$ \\ 'Museu Nacional, Departamento de Entomologia, Universidade Federal do Rio de Janeiro, Quinta da Boa Vista, São Cristóvão $20940-040$ Rio de \\ Janeiro-RJ, Brazil.vane_bio@mls.com.br
}

\begin{abstract}
Host plants of Cerambycidae and Vesperidae (Coleoptera, Chrysomeloidea) from South America. This paper offers a contribution to the knowledge on host plants of Cerambycidae and Vesperidae (Coleoptera, Chrysomeloidea) from South America. A total of 211 species of longhorn beetles belonging to 111 genera, 49 tribes, five subfamilies and two families are presented with records of host plants. Data on 259 species of host plants distributed among 188 genera and 69 families are listed with the beetle species.
\end{abstract}

KEYWORDS. Cerambycinae; host plants; Lamiinae; Leguminosae; new records.

RESUMO. Plantas-hospedeiras de Cerambycidae e Vesperidae (Coleoptera, Chrysomeloidea) da América do Sul. Uma contribuição ao conhecimento de plantas-hospedeiras das famílias Cerambycidae e Vesperidae (Coleoptera, Chrysomeloidea) da América do Sul é apresentada. Listam-se 211 espécies com registros de plantas hospedeiras, distribuídas em 111 gêneros, 49 tribos, cinco subfamílias e duas famílias. Os dados de plantas-hospedeiras associadas aos besouros totalizam 259 espécies distribuídas em 188 gêneros e 69 famílias.

PALAVRAS-CHAVE. Cerambycinae; Lamiinae; Leguminosae; novos registros; plantas-hospedeiras.

The family Cerambycidae consists of 35,000 species worldwide (Monné et al. 2009), commonly known as longhorn or timber beetles. These beetles are typically characterized by long antennae used mainly to detect pheromones, allowing mating and the localization of the appropriate host plant for oviposition (Martins 1997).

Napp (1994) recognized nine subfamilies: Anoplodermatinae, Aseminae, Cerambycinae, Lamiinae, Lepturinae, Parandrinae, Philinae, Prioninae and Spondylidinae. Svácha et al. (1997) transferred Anoplodermatinae to Vesperidae. Bouchard et al. (2011) confirmed the position of Vesperidae at family level and recognized nine subfamilies: Apatophyseinae, Cerambycinae, Dorcasominae, Lamiinae, Lepturinae, Necydalinae, Parandrinae, Prioninae and Spondylidinae.

The larvae of longhorn beetles feed on dead, dying or decaying wood, but some species feed on living plant tissue and can cause considerable economic harm (Martins 1997). For example, Phoracantha semipunctata (Fabricius, 1775) feeds on Eucalyptus sp., Hedypathes betulinus (Klug, 1825), on mate (Ilex paraguariensis Saint Hilaire, 1822), and Oncideres impluviata (Germar, 1824) on black wattle (Acacia sp.) (Monné 2001b-c, 2002a). Longhorn beetles are also fundamental to the decomposition of dead wood, incorporating plant parts to the ground and allowing the renewal of forests by opening spaces and consequent seed germination (Tavakilian et al. 1997).

The only catalogue of host plants of the families Cerambycidae and Vesperidae was printed about ten years ago (Monné 2001a-c, 2002a-b, 2004). As many papers that propose new taxa and offer new information have since been published, we have updated this catalogue of host plants of Cerambycidae and Vesperidae from South America.

\section{MATERIAL AND METHODS}

All relevant literature was extensively consulted. Host plant data sets not found in Monné (2001a-c, 2002a-b, 2004) are listed here. Host nomenclature was entirely drawn from the online checklist "The Plant List" (2010), which is joint effort of the Royal Botanic Gardens, Kew (United Kingdom) and the Missouri Botanical Garden (United States).

The taxa are listed in alphabetical order, including one introduced species marked with an asterisk. The checklist is composed only of references of hosts.

\section{RESULTS}

A total of 211 species of longhorn beetles distributed among 111 genera, 49 tribes, five subfamilies and two families are recorded. Vesperidae, with the smallest number of records, has host data for only two species of Anoplodermatini, subfamily Anoplodermatinae. The remaining taxa belong to Cerambycidae.

\section{CERAMBYCIDAE CERAMBYCINAE}

Achrysonini

Achryson maculatum Burmeister, 1865

Di Iorio, 2006: 162

Host-LEGUMINOSAE - Acacia caven (Molina) Molina 
Achryson peracchii Martins, 1976

Martins, 2002: 139

Host-LEGUMINOSAE - Piptadenia sp.

\section{Achryson surinamum (Linnaeus, 1767)}

Di Iorio, 2006: 162; Di Iorio \& Farina, 2009: 80

Hosts-ANACARDIACEAE - Schinus molle L.; JUGLANDACEAE Carya illinoinensis (Wangenh.) K. Koch; LEGUMINOSAE - Bauhinia forficata subsp. pruinosa (Vogel) Fortunato \& Wunderlin, Prosopis alpataco Phil.; MELIACEAE - Melia azedarach L.; MORACEAE - Ficus carica L.; RHAMNACEAE - Colletia paradoxa (Spreng.) Escal.; TAMARICACEAE - Tamarix gallica L.; ULMACEAE - Ulmus pumila L.

\section{Achryson undulatum Burmeister 1865}

Di Iorio \& Farina, 2009: 81

Hosts-LEGUMINOSAE - Enterolobium contortisiliquum (Vell.) Morong, Gleditsia triacanthos L.; MORACEAE - Morus nigra L.

\section{Huequenia livida (Germain, 1898)}

Villacide et al., 2006: 122

Host-PINACEAE - Pinus contorta var. murrayana (Balf.) S.Watson

\section{Basipterini}

\section{Basiptera castaneipennis Thomson, 1864}

Di Iorio, 2006: 168

Host-LEGUMINOSAE - Prosopis flexuosa DC.

\section{Callidini}

Calydon submetallicum (Blanchard, 1851)

Rizzuto, 2009: 391

Hosts-LAURACEAE - Persea lingue (Miers ex Bertero) Nees; NOTHOFAGACEAE - Nothofagus antarctica (G.Forst.) Oerst., N. betuloides (Mirb.) Oerst., N. glauca (Phil.) Krasser; WINTERACEAE Drimys winteri J. R. Forst. \& G.Forst.

\section{Cerambycini}

\section{Sphallenum tuberosum Bates, 1870}

Zanuncio et al., 2005: 339

Hosts-MALPIGHIACEAE - Stigmaphyllon sp.; MYRTACEAE - Eucalyptus cloeziana F.Muell., E. grandis W.Hill.

\section{Clytini}

Megacyllene (Megacyllene) nebulosa

(Laporte \& Gory, 1836)

Di Iorio, 2006: 165

Hosts-LEGUMINOSAE - Geoffroea decorticans (Hook. \& Arn.) Burkart, Prosopis flexuosa DC.

\section{Megacyllene (Megacyllene) acuta (Germar, 1821)}

Di Iorio \& Farina, 2009: 87

Hosts-CANNABACEAE - Celtis ehrenbergiana (Klotzsch) Liebm.; JUGLANDACEAE - Carya illinoinensis (Wangenh.) K.Koch.

\section{Neoclytus ypsilon Chevrolat, 1862}

Di Iorio, 2006: 166; Di Iorio \& Farina, 2009: 87

Hosts-ALTINGIACEAE - Liquidambar styraciflua L.; ANACARDIACEAE - Schinus myrtifolia (Griseb.) Cabrera; FAGACEAE - Quercus robur L.; JUGLANDACEAE - Carya illinoinensis (Wangenh.) K.Koch; LEGUMINOSAE - Gleditsia triacanthos L.; MORACEAE - Morus alba L, Morus nigra L.; ROSACEAE - Mespilus germanica L.

\section{Compsocerini}

\section{Aglaoschema albicorne (Fabricius, 1801)}

Dalens et al., 2010: 94

Hosts-LEGUMINOSAE - Inga sp., Pseudopiptadenia suaveolens (Miq.) J. W. Grimes

\section{Aglaoschema quieci}

Dalens, Tavakilian, \& Touroult, 2010

Dalens et al., 2010: 88

Host-LEGUMINOSAE - Vouacapoua americana Aubl.

\section{Compsocerus violaceus (White, 1853)}

Di Iorio, 2006: 163; Di Iorio \& Farina, 2009: 82

Hosts-ANACARDIACEAE - Schinopsis balansae Engl., Schinus molle L.; CELASTRACEAE - Euonymus japonicus Thunb.; FAGACEAE - Castanea sativa Mill., Quercus robur L.; JUGLANDACEAE - Carya illinoinensis (Wangenh.) K. Koch; LAURACEAE - Laurus nobilis L.; LEGUMINOSAE - Acacia dealbata Link, A. melanoxylon R. Br., A. visco Griseb., Anadenanthera colubrina var. cebil (Griseb.) Altschul, Bauhinia forficata subsp. pruinosa (Vogel) Fortunato \& Wunderlin, Calliandra tweedii Benth., Cercis siliquastrum L., Mimosa polycarpa var. spegazzinii Burkart, Prosopis affinis Spreng., P. nigra Hieron., Sesbania virgata (Cav.) Pers.; LYTHRACEAE - Punica granatum L.; OLEACEAE - Ligustrum lucidum W.T.Aiton; PINACEAE - Pinus sp.; ROSACEAE - Cerasus vulgaris Mill., Crataegus sp., Mespilus germanica L., Prunus domestica L., Rosa sp.; RUTACEAE - Citrus aurantium L., C. limon (L.) Burm. f., C. sinensis (L.) Osbeck; SALICACEAE - Salix fragilis L.; SAPINDACEAE - Dodonaea viscosa Jacq.; SAPOTACEAE - Chrysophyllum gonocarpum (Mart. \& Eichler ex Miq.) Engl.; ULMACEAE - Ulmus pumila L.

\section{Ectenessini}

\section{Eurymerus eburioides Audinet-Serville 1833}

Di Iorio \& Farina, 2009: 81

Host-CANNABACEAE - Celtis ehrenbergiana (Klotzsch) Liebm.

\section{Elaphidiini}

Curtomerus flavus (Fabricius, 1775)

Morris, 2002: 210

Host-ACANTHACEAE - Avicennia germinans. (L.) L.

\section{Eurysthea hirca (Berg, 1889)}

Di Iorio \& Farina, 2009: 81

Host-LEGUMINOSAE - Acacia visco Griseb.

Paranyssicus tresorensis Dalens, 2011

Dalens, 2011: 32

Host-ANACARDIACEAE - Anacardium sp. 
Hesperophanini

Ochrus ornatus (Fisher, 1935)

Chalumeau \& Touroult, 2005: 85

Host-LAURACEAE - Ocotea sp.

\section{Heteropsini}

Erythrochiton jucundum (Gounelle, 1913)

Di Iorio \& Farina, 2009: 87

Host-LEGUMINOSAE - Acacia caven (Molina) Molina

\section{Hexoplonini}

Gnomidolon varians Gounelle, 1909

Di Iorio, 2006: 166

Hosts-BIGNONIACEAE - Arrabidaea sp., Dolichandra unguis-cati (L.) L.G.Lohmann.

\section{Tapuruia felisbertoi Lane, 1973}

Martins, 2006: 53; Casari \& Steffanello, 2010: 425

Host-EUPHORBIACEAE - Hevea brasiliensis (Willd. ex A.Juss.) Müll.Arg.

\section{Tetraibidion aurivillii (Gounelle, 1909)}

Di Iorio, 2006: 167

Host-BIGNONIACEAE - Arrabidaea sp.

\section{Methiini}

Methia fischeri Melzer, 1923

Di Iorio, 2006: 161

Host-LEGUMINOSAE - Acacia aroma Hook. \& Arn.

\section{Nathriini}

Nathrius brevipennis (Mulsant, 1839)

Di Iorio \& Farina, 2009: 86

Hosts-MORACEAE - Morus sp.; PASSIFLORACEAE - Passiflora caerulea L.

\section{Neoibidionini}

\section{Compsa nebulosa Martins, 1970}

Di Iorio, 1997: 154

Host-SAPINDACEAE - Serjania foveata Griseb.

\section{Compsibidion fairmairei (Thomson, 1865)}

Di Iorio \& Farina, 2009: 86

Host-SAPINDACEAE - Cardiospermum grandiflorum Sw.

\section{Heterachthes flavicornis (Thomson, 1865)}

Di Iorio \& Farina, 2009: 86

Hosts-FAGACEAE - Quercus palustris Münchh., Q. robur L.; JUGLANDACEAE - Carya illinoinensis (Wangenh.) K. Koch; LEGUMINOSAE - Acacia melanoxylon R. Br., Anadenanthera colubrina var. cebil
(Griseb.) Altschul, Mimosa polycarpa var. spegazzinii Burkart; MELIACEAE - Melia azedarach L.; MORACEAE - Ficus carica L.; MYRTACEAE - Acca sellowiana (O.Berg) Burret; ROSACEAE - Mespilus germanica L.; SALICACEAE - Salix fragilis L.

\section{Heterachthes plagiatus (Burmeister, 1865)}

Di Iorio \& Farina, 2009: 86

Hosts-CANNABACEAE - Celtis australis L.; LEGUMINOSAE Acacia visco Griseb.; RHAMNACEAE - Colletia paradoxa (Spreng.) Escal.; SAPINDACEAE - Dodonaea viscosa Jacq.

\section{Heterachthes tenellus (Burmeister, 1865)}

Di Iorio, 2006: 166

Host-ANACARDIACEAE - Schinus myrtifolia (Griseb.) Cabrera

\section{Heterachthes xenocerus Martins 1960}

Di Iorio \& Farina, 2009: 87

Host-RHAMNACEAE - Colletia paradoxa (Spreng.) Escal.

Neocompsa cylindricollis (Fabricius, 1798)

Touroult, 2004: 130; Chalumeau \& Touroult, 2005: 109

Hosts-ANACARDIACEAE - Mangifera indica L.; COMBRETACEAE - Conocarpus erectus L.; ELAEOCARPACEAE - Sloanea macrophylla Spruce ex Benth.; EUPHORBIACEAE - Croton flavens L., Hippomane mancinella L.; LEGUMINOSAE - Acacia cornigera (L.) Willd., A. tortuosa (L.) Willd., Hymenaea courbaril L., Inga ingoides (Rich.) Willd., I. laurina (Sw.) Willd., Leucaena leucocephala (Lam.) de Wit, Lonchocarpus punctatus Kunth., Pithecellobium unguis-cati (L.) Benth., Tamarindus indica L.; MORACEAE - Ficus sp.

\section{Sydax stramineus Lacordaire, 1868}

Di Iorio, 2006: 161

Host-LEGUMINOSAE - Acacia nitidifolia Speg.

\section{Obriini}

\section{Obrium multifarium Berg, 1889}

Di Iorio, 1994: 21; 1997: 152, 153, 154; González \& Di Iorio, 1997: 172, 173; Di Iorio, 2006: 167

Hosts-CANNABACEAE - Celtis ehrenbergiana (Klotzsch) Liebm., C. iguanaea (Jacq.) Sarg.; CUCURBITACEAE - Siolmatra brasiliensis (Cogn.) Baill.; RHAMNACEAE - Ziziphus mistol Griseb.

\section{Obrium trifasciatum Bosq, 1951}

Di Iorio, 1994: 21; González \& Di Iorio, 1997: 172

Host-RHAMNACEAE - Ziziphus mistol Griseb.

\section{Obrium vicinum Gounelle, 1909}

Duffy, 1960: 141; Di Iorio, 1997: 152

Hosts-CUCURBITACEAE - Siolmatra brasiliensis (Cogn.) Baill.; PODOCARPACEAE - Podocarpus andina Poepp. ex Endl.

\section{Oemini}

\section{Temnopis martinezi Martins, 1985}

Di Iorio, 2006: 161

Hosts-LEGUMINOSAE - Acacia aroma Hook. \& Arn., Prosopis alba Griseb., P. torquata (Lag.) DC. 


\section{Rhinotragini \\ Acatinga gallardi}

(Peñaherrera-Leiva \& Tavakilian, 2004)

Peñaherrera-Leiva \& Tavakilian, 2004: 126; Tavakilian \& Peñaherrera-Leiva, 2007: 100

Host-CELASTRACEAE - Maytenus sp.

\section{Carenoptomerus guyanensis}

Tavakilian \& Peñaherrera-Leiva, 2003

Tavakilian \& Peñaherrera-Leiva, 2003a: 280; Tavakilian \& PeñaherreraLeiva, 2007: 81

Host-SAPOTACEAE - Ecclinusa guianensis Eyma

Chrysaethe jorgei

(Tavakilian \& Peñaherrera-Leiva, 2003)

Tavakilian \& Pañaherrera-Leiva, 2003a: 305; 2007: 94

Host-ANNONACEAE - Oxandra asbeckii (Pulle) R.E.Fr.

\section{Chrysommata lauracea}

(Peñaherrera-Leiva \& Tavakilian, 2003)

Peñaherrera-Leiva \& Tavakilian, 2003: 193; Tavakilian \& Peñaherrera-Leiva, 2007: 94

Host-LAURACEAE - Mezilaurus ita-uba (Meisn.) Taub. ex Mez.

\section{Eclipta amanoaphila}

(Peñaherrera-Leiva \& Tavakilian, 2003)

Peñaherrera-Leiva \& Tavakilian, 2003: 201; Tavakilian \& Peñaherrera-Leiva, 2007: 97

Host-PHYLLANTHACEAE - Amanoa guianensis Aubl.

\section{Eclipta bauhiniae}

(Peñaherrera-Leiva \& Tavakilian, 2004)

Peñaherrera-Leiva \& Tavakilian, 2004: 138; Tavakilian \& Peñaherrera-Leiva, 2007: 97

Host-LEGUMINOSAE - Bauhinia guianensis Aubl.

\section{Eclipta eperuaphila}

(Tavakilian \& Peñaherrera-Leiva, 2005)

Tavakilian \& Peñaherrera-Leiva, 2005: 31

Host-LEGUMINOSAE - Eperua rubiginosa Miq.

\section{Eclipta fanchonae}

(Tavakilian \& Peñaherrera-Leiva, 2003)

Tavakilian \& Peñaherrera-Leiva, 2003a: 293

Host-LEGUMINOSAE - Zygia racemosa (Ducke) Barneby \& J. W. Grimes.

\section{Eclipta taraleaphila}

(Tavakilian \& Peñaherrera-Leiva, 2003)

Tavakilian \& Peñaherrera-Leiva, 2003a: 294

Host-LEGUMINOSAE - Taralea oppositifolia Aubl.
Ecliptoides rouperti

(Tavakilian \& Peñaherrera-Leiva, 2005)

Tavakilian \& Peñaherrera-Leiva, 2005: 38

Hosts-LEGUMINOSAE - Ormosia nobilis Tul., Vatairea paraensis Ducke.

\section{Epimelitta durantoni}

Peñaherrera-Leiva \& Tavakilian, 2003

Peñaherrera-Leiva \& Tavakilian, 2003: 206

Host-LEGUMINOSAE - Pseudopiptadenia suaveolens (Miq.) J. W. Grimes.

\section{Etimasu cosmipes}

(Peñaherrera-Leiva \& Tavakilian, 2003)

Peñaherrera-Leiva \& Tavakilian, 2003: 183

Hosts-LAURACEAE - Aniba parviflora (Meisn.) Mez, Licaria chrysophylla (Meisn.) Kosterm., Misanteca martiniana (Mez) Lundell.

\section{Ischasia ecclinusae}

Peñaherrera-Leiva \& Tavakilian, 2004

Peñaherrera-Leiva \& Tavakilian, 2004: 120

Host-SAPOTACEAE - Ecclinusa guianensis Eyma.

\section{Ischasia feuilleti}

Peñaherrera-Leiva \& Tavakilian, 2003

Peñaherrera-Leiva \& Tavakilian, 2003: 194

Host-SAPOTACEAE - Chrysophyllum sanguinolentum (Pierre) Baehni

\section{Ischasia mareki}

Peñaherrera-Leiva \& Tavakilian, 2004

Peñaherrera-Leiva \& Tavakilian, 2004: 124

Hosts-SAPOTACEAE - Chrysophyllum lucentifolium Cronquist, C. prieurii A. DC., Ecclinusa guianensis Eyma, Micropholis guyanensis (A DC.) Pierre, M. obscura T. D. Penn., Pouteria cayennensis (A.DC.) Eyma, P. oblanceolata Pires.

\section{Ischasia pouteriae}

Peñaherrera-Leiva \& Tavakilian, 2004

Peñaherrera-Leiva \& Tavakilian, 2004: 123

Host-SAPOTACEAE - Pouteria sp.

\section{Ischasia sabatieri}

Peñaherrera-Leiva \& Tavakilian, 2004

Peñaherrera-Leiva \& Tavakilian, 2004: 122

Host-SAPOTACEAE-Micropholis venulosa (Mart. \& Eichler ex Miq.) Pierre.

\section{Ischasia viridithorax}

Peñaherrera-Leiva \& Tavakilian, 2004

Peñaherrera-Leiva \& Tavakilian, 2004: 121

Host-SAPOTACEAE - Ecclinusa guianensis Eyma.

Odontocera annulicornis Magno, 2001

Tavakilian \& Peñaherrera-Leiva, 2003a: 286

Hosts-LEGUMINOSAE - Bocoa prouacensis Aubl., Chamaecrista 
apoucouita (Aubl.) H. S. Irwin \& Barneby, Crudia aromatica (Aubl.) Willd., C. bracteata Benth., Swartzia panacoco (Aubl.) Cowan, S. polyphylla DC., $S$. remiger Amshoff.

\section{Odontocera barnouini}

Peñaherrera-Leiva \& Tavakilian, 2003

Peñaherrera-Leiva \& Tavakilian, 2003: 171

Host-POLYGONACEAE - Coccoloba ascendens Duss ex Lindau.

\section{Odontocera flavicauda Bates 1873}

Di Iorio \& Farina, 2009: 85

Hosts-RHAMNACEAE - Colletia paradoxa (Spreng.) Escal.; SAPINDACEAE - Dodonaea viscosa Jacq.

\section{Odontocera javieri}

Tavakilian \& Peñaherrera-Leiva, 2003

Tavakilian \& Peñaherrera-Leiva, 2003a: 287

Hosts-LEGUMINOSAE - Dipteryx punctata (S.F.Blake) Amshoff, Taralea oppositifolia Aubl

\section{Odontocera morii}

Tavakilian \& Peñaherrera-Leiva, 2003

Tavakilian \& Peñaherrera-Leiva, 2003a: 289

Host-LEGUMINOSAE - Ormosia nobilis Tul

\section{Odontocera nigrovittata}

Tavakilian \& Peñaherrera-Leiva, 2003

Tavakilian \& Peñaherrera-Leiva, 2003a: 283

Host-CONNARACEAE - Connarus sp.

\section{Odontocera quiinaphila}

Peñaherrera-Leiva \& Tavakilian, 2003

Peñaherrera-Leiva \& Tavakilian, 2003: 167

Host-OCHNACEAE - Quiina oiapocensis Pires.

\section{Odontocera sabatieri}

Tavakilian \& Peñaherrera-Leiva, 2003

Tavakilian \& Peñaherrera-Leiva, 2003a: 289

Host-LEGUMINOSAE - Inga alba (Sw.) Willd.

\section{Ommata elegans White, 1855}

Tavakilian \& Peñaherrera-Leiva, 2007: 100

Host-SAPINDACEAE - Serjania brachycarpa A. Gray ex Radlk.

\section{Phygopoda ingae}

Peñaherrera-Leiva \& Tavakilian, 2004

Peñaherrera-Leiva \& Tavakilian, 2004: 143

Host-LEGUMINOSAE - Inga alba (Sw.) Willd.

\section{Phygopoides pradosiae}

\section{Peñaherrera-Leiva \& Tavakilian, 2003}

Peñaherrera-Leiva \& Tavakilian, 2003: 187

Hosts-SAPOTACEAE - Pouteria cayennensis (A.DC.) Eyma, Pradosia cochlearia (Lecomte) T. D. Penn.
Rhopalessa clavicornis (Bates, 1873)

Di Iorio, 2006: 164; Di Iorio \& Farina, 2009: 85

Hosts-LEGUMINOSAE - Acacia bonariensis Hook. \& Arn., A. nitidifolia Speg.; MELIACEAE - Melia azedarach L.

\section{Rhopalophorini}

Dihammaphora bruchi Aurivillius, 1922

Di Iorio, 2006: 165

Hosts-CANNABACEAE - Celtis ehrenbergiana (Klotzsch) Liebm., C. iguanaea (Jacq.) Sarg.; MORACEAE - Morus nigra L.

\section{Dihammaphora signaticollis Chevrolat, 1859}

Galileo \& Martins, 2006: 164

Host-FAGACEAE - Castanea sativa Mill.

Dirocoremia bruchi (Gounelle, 1905)

Di Iorio, 2006: 164

Hosts-LEGUMINOSAE - Prosopis flexuosa DC., P. torquata (Lag.) DC.

Ischionodonta platensis (Chevrolat, 1859)

Di Iorio, 2006: 164; Di Iorio \& Farina, 2009: 87

Host-CANNABACEAE - Celtis ehrenbergiana (Klotzsch) Liebm.

\section{Smodicini}

*Smodicum cucujiforme (Say, 1826)

MacRae \& Rice, 2007: 244

Hosts-FAGACEAE - Quercus velutina Lam.; ULMACEAE - Ulmus rubra Muhl.

\section{Trachyderini}

Andraegoidus rufipes (Fabricius, 1787)

Fernandes \& Santos, 2009: 155

Host-VERBENACEAE - Lantana camara L.

Chydarteres striatus striatus (Fabricius, 1787)

Di Iorio \& Farina, 2009: 88

Hosts-CASUARINACEAE - Casuarina cunninghamiana Miq.; JUGLANDACEAE - Carya illinoinensis (Wangenh.) K.Koch; LEGUMINOSAE - Acacia caven (Molina) Molina, Gleditsia triacanthos L.; MORACEAE - Morus nigra L.; SALICACEAE - Salix babylonica L.; SAPINDACEAE - Dodonaea viscosa Jacq.

Dorcacerus barbatus (Olivier, 1790)

Di Iorio, 2006: 167; Fernandes \& Santos, 2009: 155

Hosts-LEGUMINOSAE - Prosopis flexuosa DC.; VERBENACEAE - Lantana camara L.

Eriocharis richardii (Dupont, 1838)

Di Iorio, 2006: 167; Di Iorio \& Farina, 2009: 88

Hosts-LEGUMINOSAE - Gleditsia triacanthos L., Prosopis alpataco Phil.

Oxymerus aculeatus lebasii Dupont, 1838

Touroult, 2004: 130; Chalumeau \& Touroult; 2005: 133; Fonseca et al., 2008: 510 
Hosts-COMBRETACEAE - Conocarpus erectus L.; LEGUMINOSAE - Albizzia sp., Inga ingoides (Rich.) Willd., I. laurina (Sw.) Willd., Mimosa pudica L.; MORACEAE - Artocarpus altilis (Parkinson ex F.A.Zorn) Fosberg; SOLANACEAE - Solanum erianthum D. Don.

\section{Oxymerus basalis (Dalman, 1823)}

Zanuncio et al., 2009: 179

Host-MYRTACEAE - Eucalyptus sp.

\section{Oxymerus luteus australis Huedepohl 1979}

Di Iorio \& Farina, 2009: 88

Hosts-LEGUMINOSAE - Gleditsia triacanthos L., Parkinsonia aculeata L.; ROSACEAE - Pyrus communis L.

Oxymerus pallidus Dupont, 1838

Di Iorio, 2006: 167

Host-SAPOTACEAE - Sideroxylon obtusifolium subsp. Obtusifolium

Oxymerus virgatus Gounelle, 1913

Di Iorio, 2006: 167

Host-LEGUMINOSAE - Geoffroea decorticans (Hook. \& Arn.) Burkart.

Retrachydes thoracicus sulcatus (Burmeister, 1865)

Di Iorio, 2006: 168

Hosts-LEGUMINOSAE - Acacia furcatispina Burkart, A. visco Griseb.

Retrachydes thoracicus thoracicus (Olivier, 1790)

Monné et al., 2002: 67

Host-SALICACEAE - Salix sp.

Trachyderes (Trachyderes) succinctus duponti Aurivillius, 1912

Fernandes \& Santos, 2009: 155

Host-VERBENACEAE - Lantana camara L.

\section{Unxiini}

Ethemon basale (Burmeister, 1865)

Di Iorio, 2006: 164

Host-ANACARDIACEAE - Schinopsis balansae Engl.

Ethemon brevicorne Napp \& Reynaud, 1998

Di Iorio, 2006: 164

Host-LEGUMINOSAE - Prosopis alba Griseb.

\section{Unxia gracilior (Burmeister, 1865)}

Monné et al., 2002: 67; Di Iorio \& Farina, 2009: 85

Hosts-MYRTACEAE - Eucalyptus camaldulensis Dehnh.; ROSACEAE - Malus sp.

\section{Paromoeocerus barbicornis (Fabricius, 1792)}

Di Iorio \& Farina, 2009: 83

Hosts-ANACARDIACEAE - Schinus molle L; CELASTRACEAE Euonymus japonicus Thunb.; EUPHORBIACEAE - Sapium haematospermum Müll.Arg.; FAGACEAE - Castanea sativa Mill., Quercus palustris Münchh., Q. robur L.; JUGLANDACEAE - Carya illinoinensis (Wangenh.)
K. Koch, Juglans regia L.; LEGUMINOSAE - Acacia caven (Molina) Molina, Anadenanthera colubrina var. cebil (Griseb.) Altschul, Gleditsia triacanthos L., Mimosa polycarpa var. spegazzinii Burkart, Prosopis torquata (Lag.) DC. Sesbania virgata (Cav.) Pers.; LYTHRACEAE - Punica granatum L.; MORACEAE - Ficus carica L.; ROSACEAE - Mespilus germanica L., Rosa sp.; RUTACEAE - Citrus limon (L.) Burm. f.; ULMACEAE - Ulmus pumila L.

\section{LAMIINAE}

Acanthocinini

Baryssiniella hieroglyphica Berkov \& Monné, 2010

Berkov \& Monné, 2010: 54

Hosts-LECYTHIDACEAE - Bertholletia excelsa Bonpl., Eschweilera coriacea (DC.) S.A.Mori.

Baryssiniella tavakiliani Berkov \& Monné, 2010

Berkov \& Monné, 2010: 57

Hosts-LECYTHIDACEAE - Corythophora amapaensis Pires ex S.A.Mori \& Prance, Eschweilera parviflora (Aubl.) Miers.

\section{Eutrypanus dorsalis (Germar, 1824)}

Di Iorio \& Farina, 2009: 89

Host-MELIACEAE - Melia azedarach L.

\section{Hyperplatys argentina (Berg, 1889)}

Di Iorio, 2004: 53; Di Iorio \& Farina, 2009: 89

Hosts-APOCYNACEAE - Araujia hortorum E.Fourn., Morrenia odorata (Hook. \& Arn.) Lindl.; MORACEAE - Ficus carica L., Maclura pomifera (Raf.) C. K. Schneid.

\section{Leptocometes virescens (Melzer, 1931)}

Mecke, 2002: 30

Host-ARAUCARIACEAE - Araucaria angustofolia (Bertol.) Kuntze.

Leptostylus perniciosus Monné \& Hoffmann, 1981

Mecke, 2002: 26

Host-RUTACEAE - Citrus sinensis (L.) Osbeck.

Lophopoeum bruchi Monné \& Martins, 1976

Di Iorio, 2004: 53

Hosts-LEGUMINOSAE - Acacia aroma Hook. \& Arn., Enterolobium contortisiliquum (Vell.) Morong, Prosopis sp., P. caldenia Burkart, P. hassleri Harms, P. kuntzei Kuntze.

Lophopoeum timbouvae Lameere, 1884

Di Iorio, 2004: 54; Di Iorio \& Farina, 2009: 90

Hosts-LEGUMINOSAE - Acacia caven (Molina) Molina, Gleditschia triacanthos L.

Nealcidion bicristatum (Bates, 1863)

Olckers et al., 2002: 257; Di Iorio, 2004: 54

Host-SOLANACEAE - Solanum mauritianum Scop.

Nealcidion cereicola (Fisher, 1936)

Di Iorio, 2004: 55

Hosts-CACTACEAE - Cereus hildmannianus K.Schum., C. respandus (L.) Mill., C. spegazzinii F. A. C. Weber, Echinopsis schickendantzii F. A. 
C. Weber; MORACEAE - Ficus carica L.

Neobaryssinus altissimus Berkov \& Monné, 2010

Berkov \& Monné, 2010: 51

Host-LECYTHIDACEAE - Lecythis poiteaui O.Berg.

Oectropsis franciscae Barriga \& Cepeda, 2005

Barriga \& Cepeda, 2005: 48

Hosts-NOTHOFAGACEAE - Nothofagus alessandrii Espinosa, N. alpina (Poepp. \& Endl.) Oerst., N. dombeyi (Mirb.) Oerst., N. obliqua (Mirb.) Oerst.

\section{Trypanidius andicola Blanchard, 1846}

Roddick et al., 2009: 105

Host-EUPHORBIACEAE - Hura crepitans L.

Trypanidius notatus (Fabricius, 1787)

Roddick et al., 2009: 105

Host-EUPHORBIACEAE - Hura crepitans L.

\section{Urgleptes bruchi (Melzer, 1932)}

Di Iorio, 2004: 56; Di Iorio \& Farina, 2009: 90

Hosts-APOCYNACEAE - Araujia hortorum E.Fourn.; LEGUMINOSAE - Spartium junceum L.; MORACEAE - Ficus carica L., Maclura pomifera (Raf.) C.K.Schneid.; OLEACEAE - Ligustrum sinense Lour.

\section{Urgleptes mancus (Melzer, 1932)}

Di Iorio \& Farina, 2009: 90

Hosts-MALVACEAE - Hibiscus syriacus L.; MORACEAE - Ficus carica L.; PASSIFLORACEAE - Passiflora caerulea L.; SOLANACEAE - Solanum glaucophyllum Desf.

\section{Acanthoderini}

Dryoctenes scrupulosus (Germar, 1824)

Di Iorio, 2004: 58

Hosts-MALVACEAE - Ceiba insignis (Kunth) P. E. Gibbs \& Semir, C. pubiflora (A. St.-Hil.) K. Schum.; MORACEAE - Ficus carica L.

\section{Exalphus guaraniticus (Lane, 1955)}

Di Iorio, 2004: 58

Host-MORACEAE - Maclura tinctoria (L.) D.Don ex Steud.

Macropophora accentifer (Olivier, 1795)

Di Iorio, 2004: 61

Hosts-EUPHORBIACEAE - Vernicia fordii (Hemsl.) Airy Shaw; RUTACEAE - Balfourodendron riedelianum (Engl.) Engl.

\section{Nesozineus obscurus Hoffmann, 1984}

Di Iorio, 2004: 59

Host-LEGUMINOSAE - Prosopis flexuosa DC.

Oreodera aerumnosa Erichson, 1847

Tavakilian \& Néouze, 2011: 97

Host-MORACEAE - Morus nigra L.

\section{Oreodera bituberculata Bates, 1861}

Tavakilian \& Néouze, 2011: 93
Hosts-APOCYNACEAE - Couma guianensis Aubl.; CLUSIACEAE - Clusia scrobiculata Benoist; OLACACEAE - Chaunochiton kappleri (Sagot ex Engl.) Ducke.

\section{Oreodera crinita Monné \& Fragoso, 1988}

Tavakilian \& Néouze, 2011: 80

Host-LEGUMINOSAE - Eperua rubiginosa Miq.

Oreodera griseozonata Bates, 1861

Tavakilian \& Néouze, 2011: 81

Host-MORACEAE - Bagassa guianensis Aubl.

\section{Oreodera neglecta Melzer, 1932}

Tavakilian \& Néouze, 2011: 65

Host-BURSERACEAE - Protium aracouchini Marchand

Oreodera olivosimplex Néouze \& Tavakilian, 2010

Néouze \& Tavakilian, 2010: 61; Tavakilian \& Néouze, 2011: 68

Hosts-CARYOCARACEAE - Caryocar microcarpum Ducke; COMBRETACEAE - Buchenavia nitidissima (Rich.) Alwan \& Stace.

\section{Oreodera sororcula Martins \& Monné, 1993}

Tavakilian \& Néouze, 2011: 84

Host-CLUSIACEAE - Symphonia globulifera L. f.

Penaherreraus guianensis

(Tavakilian \& Peñaherrera-Leiva, 2003)

Tavakilian \& Peñaherrera-Leiva, 2003b: 10

Hosts-SAPOTACEAE - Ecclinusa guianensis Eyma, Micropholis guyanensis (A. DC.) Pierre.

\section{Penaherreraus pradosiae}

(Tavakilian \& Peñaherrera-Leiva, 2003)

Tavakilian \& Peñaherrera-Leiva, 2003b: 13

Hosts-SAPOTACEAE - Pouteria sp., Pradosia cochlearia (Lecomte) T. D. Penn., P. ptychandra (Eyma) T. D. Penn.

\section{Penaherreraus sarryi}

(Tavakilian \& Peñaherrera-Leiva, 2003)

Tavakilian \& Peñaherrera-Leiva, 2003b: 12

Hosts-SAPOTACEAE - Chrysophyllum sanguinolentum sanguinolentum, Micropholis guyanensis (A. DC.) Pierre, Pouteria cayennensis (A. DC.) Eyma.

\section{Psapharochrus jaspideus (Germar, 1824)}

Di Iorio, 2004: 56; Di Iorio \& Farina, 2009: 90

Hosts-ADOXACEAE - Sambucus australis Cham. \& Schltdl.; ALTINGIACEAE - Liquidambar styraciflua L.; ANACARDIACEAE Schinus molle L.; BETULACEAE - Betula pubescens var. pubescens; BIGNONIACEAE - Handroanthus impetiginosus (Mart. ex DC.) Mattos, Jacaranda mimosifolia D.Don; COMPOSITAE - Baccharis dracunculifolia DC.; CONVOLVULACEAE - Ipomoea cairica (L.) Sweet; EUPHORBIACEAE - Euphorbia pulcherrima Willd. ex Klotzsch, Manihot esculenta Crantz; JUGLANDACEAE - Carya illinoinensis (Wangenh.) K.Koch; LEGUMINOSAE - Acacia visco Griseb., Bauhinia forficata subsp. pruinosa (Vogel) Fortunato \& Wunderlin, Geoffroea decorticans (Hook. \& Arn.) Burkart, Gleditsia triacanthos L., Prosopis alba Griseb, P. nigra Hieron., 
Robinia pseudoacacia L., Spartium junceum L., Styphnolobium japonicum (L.) Schott; LORANTHACEAE - Tripodanthus acutifolius (Ruiz \& Pav.) Tiegh.; MALVACEAE - Hibiscus mutabilis L., H. rosa-sinensis L., $H$. syriacus L.; MELIACEAE - Melia azedarach L.; OLEACEAE - Fraxinus americana L.; PINACEAE - Pinus patula Schiede ex Schltdl. \& Cham., Pinus taeda L.; ROSACEAE - Mespilus germanica L.; RUTACEAE - Citrus aurantium L., C. limon (L.) Burm. f., C. sinensis (L.) Osbeck; SALICACEAE - Populus nigra L., Salix humboldtiana Willd.; SAPINDACEAE - Acer saccharinum L.; ULMACEAE - Ulmus pumila L.

\section{Psapharochrus longispinis (Bates, 1861)}

Abreu et al., 2009: 432

Host-MALVACEAE - Scleronema micranthum (Ducke) Ducke

\section{Pycnomorphidiellus polyphagus}

Tavakilian \& Peñaherrera-Leiva, 2003

Tavakilian \& Peñaherrera-Leiva, 2003b: 16

Hosts-CHRYSOBALANACEAE - Licania cyathodes Benoist; LEGUMINOSAE - Vouacapoua americana Aubl.; PERACEAE - Chaetocarpus schomburgkianus (Kuntze) Pax \& K.Hoffm.

\section{Steirastoma breve (Sulzer, 1776)}

Di Iorio, 2004: 60

Host-SALICACEAE - Salix humboldtiana Willd.

\section{Steirastoma histrionica White, 1855}

Vitali et al., 2006

Hosts-MALVACEAE - Ceiba pentandra (L.) Gaertn, Pachira quinata (Jacq.) W. S. Alverson, Sterculia apetala (Jacq.) H. Karst.

\section{Steirastoma marmoratum (Thunberg, 1822)}

Mecke, 2002: 28

Host-MYRTACEAE - Eucalyptus sp.

Steirastoma melanogenys White, 1855

Abreu et al., 2009: 432

Host-MALVACEAE - Scleronema micranthum (Ducke) Ducke.

\section{Acrocinini}

Acrocinus longimanus (Linnaeus, 1758)

Di Iorio, 2004: 61

Host-MORACEAE - Maclura tinctoria (L.) D.Don ex Steud.

\section{Aerenicini}

Phaula thomsoni Lacordaire, 1872

Di Iorio, 2004: 64

Host-BIGNONIACEAE - Jacaranda mimosifolia D.Don.

\section{Agapanthiini}

\section{Hippopsis (Hippopsis) pertusa}

Galileo \& Martins, 1988

Di Iorio, 2004: 64

Hosts-COMPOSITAE - Xanthium cavanillesii Schouw; LEGUMINOSAE - Medicago sativa L.

\author{
Anisocerini \\ Anisocerus scopifer (Germar, 1824)
}

Di Iorio, 2004: 65

Host-LEGUMINOSAE - Mimosa bimucronata (DC.) Kuntze.

Apomecynini

Adetus similis Bruch, 1939

Di Iorio, 2004: 65

Host-CUCURBITACEAE - Cayaponia bonariensis (Mill.) Mart.Crov.

Asyngenes (Asyngenes) chalceolus Bates, 1880

Duffy, 1960: 271; Di Iorio \& Farina, 2009: 93

Host-MORACEAE - Ficus carica L.

\section{Bebelis picta Pascoe 1875}

Di Iorio \& Farina, 2009: 94

Host-CUCURBITACEAE - Cayaponia bonariensis (Mill.) Mart.Crov.

Bisaltes (Bisaltes) montevidensis (Thomson, 1868)

Di Iorio, 2004: 65

Host-SOLANACEAE - Solanum glaucophyllum Desf.

\section{Colobotheini}

Colobothea berkovi Monné \& Monné, 2010

Monné \& Monné, 2010: 42

Host-LECYTHIDACEAE - Gustavia augusta L.

Colobothea fasciata Bates, 1865

Di Iorio, 2004: 66

Host-MORACEAE - Ficus sp.

Colobothea musiva (Germar, 1824)

Di Iorio, 2004: 66

Hosts-LEGUMINOSAE - Mimosa bimucronata (DC.) O. Kuntze; RUTACEAE - Balfourodendron riedelianum (Engl.) Engl.

Colobothea poecila (Germar, 1824)

Di Iorio, 2004: 66

Hosts-LEGUMINOSAE - Mimosa bimucronata (DC.) O. Kuntze; RUTACEAE - Balfourodendron riedelianum (Engl.) Engl.

\section{Compsosomatini}

Aerenea quadriplagiata (Boheman, 1859)

Di Iorio, 2004: 66

Hosts-COMPOSITAE - Xanthium cavanillesii Schouw; MYRTACEAE - Eucalyptus viminalis Labill.

\section{Compsosoma phaleratum Thomson, 1857}

Giacomel, 1979: 98

Hosts-ANACARDIACEAE - Schinus sp., S. terebinthifolius Raddi; CLETHRACEAE - Clethra scabra Pers.; ERYTHROXYLACEAE - 
Erythroxylum havanense Jacq.; MALVACEAE - Luehea divaricata Mart; RHAMNACEAE - Scutia buxifolia Reissek; SAPINDACEAE - Allophylus edulis (A. St.-Hil., A. Juss. \& Cambess.) Radlk, Cupania vernalis Cambess., Matayba elaeagnoides Radlk

\section{Desmiphorini}

Cicatrisestola humeralis Martins \& Galileo, 1995

Di Iorio, 2004: 66

Host-CANNABACEAE - Celtis iguanaea (Jacq.) Sarg.

Desmiphora (Desmiphora) cirrosa Erichson, 1847

Di Iorio, 2004: 66

Hosts-BIGNONIACEAE - Dolichandra cynanchoides Cham.; BORAGINACEAE - Cordia americana (L.) Gottschling \& J.S.Mill.

\section{Desmiphora (Desmiphora) cucullata Thomson, 1868}

Di Iorio, 2004: 66

Host-MYRTACEAE - Hexachlamys edulis (O.Berg) Kausel \& D.Legrand.

\section{Desmiphora (Desmiphora) hirticollis (Olivier, 1795)}

Di Iorio \& Farina, 2009: 92

Hosts-APOCYNACEAE - Araujia hortorum E.Fourn., Morrenia odorata (Hook. \& Arn.) Lindl.; CANNABACEAE - Celtis ehrenbergiana (Klotzsch) Liebm.; LEGUMINOSAE - Sesbania virgata (Cav.) Pers.; MALVACEAE - Hibiscus syriacus L.; MORACEAE - Maclura pomifera (Raf.) C.K.Schneid., Morus alba L.; PASSIFLORACEAE - Passiflora caerulea L.; PLATANACEAE - Platanus acerifolia (Aiton) Willd.; SOLANACEAE - Solanum glaucophyllum Desf.

\section{Desmiphora (Desmiphora) lateralis Thomson, 1868}

Marinoni, 1969: 470

Host-MYRTACEAE - Blepharocalyx sp.

\section{Desmiphora (Desmiphora) santossilvai}

Galileo \& Martins, 2003

Tavakilian \& Néouze, 2004: 63

Hosts-SAPOTACEAE - Chrysophyllum sanguinolentum (Pierre) Baehni, Manilkara bidentata (A. DC.) A.Chev., Micropholis guyanensis (A. DC.) Pierre.

\section{Estola albocincta Melzer, 1932}

Di Iorio, 2004: 67

Host-MORACEAE - Ficus sp.

Gibbestoloides compacta Breuning, 1940

Di Iorio, 1994: 17; Di Iorio, 2004: 67; Di Iorio, 2006: 171

Host-BIGNONIACEAE - Fridericia dichotoma (Jacq.) L.G. Lohmann.

\section{Hemilophini}

Apagomerella versicolor (Boheman, 1859)

Logarzo et al., 2002: 22; Di Iorio, 2004: 61

Hosts-COMPOSITAE - Ambrosia scabra Hook. \& Arn., Tessaria absinthioides (Hook. \& Arn.) DC., Xanthium cavanillesii Schouw.
Phoebemima ensifera Tippmann, 1960

Clarke \& Zamaloa, 2009: 287

Hosts-LEGUMINOSAE - Albizia niopoides (Benth.) Burkart, Chloroleucon tenuiflorum (Benth.) Barneby \& J. W.Grimes, Inga sp., Samanea tubulosa (Benth.) Barneby \& J. W.Grimes.

\section{Zeale nigromaculata (Klug, 1829)}

Di Iorio, 2004: 68

Host-LAMIACEAE - Mentha arvensis var. piperascens Malinv. ex Holmes.

\section{Onciderini}

Hypsioma gibbera Audinet-Serville, 1835

Di Iorio, 2004: 68

Host-LEGUMINOSAE - Mimosa bimucronata (DC.) Kuntze.

Jamesia globifera (Fabricius, 1801)

Abreu et al., 2009: 432

Host-MALVACEAE - Scleronema micranthum (Ducke) Ducke.

Lochmaeocles callidryas (Bates, 1865)

Tavakilian et al., 1997: 326

Host-LEGUMINOSAE - Albizia pedicellaris (Dc.) L.Rico.

Lochmaeocles fasciatus (Lucas, 1857)

Di Iorio, 2004: 68

Host-LAURACEAE - Persea americana Mill.

\section{Oncideres germarii Thomson, 1868}

Di Iorio, 2004: 69; Di Iorio, 2006: 170; Ganci, 2010: 89

Hosts-LEGUMINOSAE - Acacia aroma Hook. \& Arn., A. dealbata Link, A. furcatispina Burkart, A. visco Griseb., Anadenanthera colubrina (Vell.) Brenan, Leucaena leucocephala (Lam.) de Wit, Parkinsonia aculeata L., Prosopis flexuosa DC., Robinia pseudoacacia L.

\section{Oncideres guttulata Thomson, 1868}

Di Iorio, 2004: 70; Di Iorio, 2006: 170

Hosts-LEGUMINOSAE - Acacia aroma Hook. \& Arn., Anadenanthera colubrina (Vell.) Brenan, Mimosa detinens Benth., Prosopis caldenia Burkart, P. flexuosa DC., P. strombulifera (Lam.) Benth; ULMACEAE Ulmus pumila L.

\section{Oncideres humeralis Thomson, 1868}

Paulino Neto et al., 2005: 7

Hosts-MELASTOMATACEAE - Acinodendron jucunda (DC.) Kuntze, Leandra scabra DC., Miconia latecrenata(DC.) Naudin, M. sellowiana Naudin.

\section{Oncideres impluviata (Germar, 1824)}

Paulino Neto et al., 2005: 9

Host-PRIMULACEAE - Myrsine coriacea subsp. coriacea.

\section{Oncideres miniata Thomson, 1868}

Di Iorio, 2004: 72

Host-AQUIFOLIACEAE - Ilex paraguariensis A.St.-Hil. 
Oncideres schreiteri Bruch, 1941

Di Iorio, 2004: 72

Host-LEGUMINOSAE - Acacia caven (Molina) Molina.

Oncideres stillata Aurivillius 1904

Di Iorio \& Farina, 2009: 94

Host-LEGUMINOSAE - Prosopis alba Griseb.

Plerodia syrinx (Bates, 1865)

Souza et al., 2001: 476

Host-CUCURBITACEAE - Sechium edule (Jaqc.) Sw.

\section{Parmenini}

Schreiteria bruchi Melzer, 1933

Di Iorio, 2004: 73

Host-APIACEAE - Azorella compacta Phil..

Schreiteria colombiana Monné \& Monné, 2006

Monné \& Monné, 2006: 489

Host-COMPOSITAE - Espeletia grandiflora Humb. \& Bonpl.

Pogonocherini

Lypsimena nodipennis (Burmeister, 1865)

Di Iorio, 2004: 74

Host-EUPHORBIACEAE - Manihot esculenta Crantz.

\section{Pteropliini}

Ataxia luteifrons (Bruch, 1926)

Di Iorio, 2004: 74

Host-ANACARDIACEAE - Schinopsis quebracho-colorado (Schltdl.) F.A. Barkley \& T. Mey.

Rhaphiptera avicenniae Dalens \& Tavakilian, 2007

Dalens \& Tavakilian in Tavakilian et al., 2007: 6

Host-ACANTHACEAE - Avicennia germinans (L.) L.

\section{Tapeinini}

Tapeina coronata coronata

Lepeletier \& Audinet-Serville, 1828

Silva et al., 1968: 409 Di Iorio, 2004: 74

Host-ANACARDIACEAE - Schinopsis quebracho-colorado (Schltdl.) F. A. Barkley \& T. Mey.

\section{Xenofreini}

Xenofrea berkovi Néouze \& Tavakilian, 2005

Néouze \& Tavakilian, 2005: 133

Hosts-MALVACEAE - Catostemma fragrans Benth., Eriotheca sp.

Xenofrea proxima Néouze \& Tavakilian, 2005

Néouze \& Tavakilian, 2005: 139

Host-LECYTHIDACEAE - Eschweilera micrantha (O.Berg) Miers.
Xenofrea rogueti Néouze \& Tavakilian, 2005

Néouze \& Tavakilian, 2005: 143

Host-LECYTHIDACEAE - Eschweilera coriacea (DC.) S.A.Mori.

PARANDRINAE

Parandrini

Parandra (Hesperandra) tucumana Zikán, 1948

Di Iorio, 2004: 76

Host-BETULACEAE - Alnus sp.

\section{PRIONINAE}

Anacolini

Prionapterus staphilinus Guérin-Méneville, 1831

Di Iorio, 2004: 76

Host-COMPOSITAE - Flourensia oolepis S.F.Blake.

\section{Callipogonini}

Callipogon (Enoplocerus) armillatus (Linnaeus, 1767)

Di Iorio, 2004: 76

Host-BIGNONIACEAE - Handroanthus impetiginosus (Mart. ex DC.) Mattos.

Navosoma luctuosum (Schoenherr, 1817)

Di Iorio, 2004: 76

Host-BIGNONIACEAE - Jacaranda cuspidifolia Mart.

Seticeros aquilus (Thomson, 1865)

Casari \& Santos-Silva, 2010: 746

Host-FAGACEAE - Quercus humboldtii Bonpl.

\section{Calocomini}

Calocomus desmarestii (Guérin-Méneville, 1831)

Di Iorio, 2004: 76

Host-VERBENACEAE - Aloysia gratissima (Gillies \& Hook.) Tronc.

Calocomus morosus White, 1850

Di Iorio, 2004: 76

Host-COMPOSITAE - Flourensia oolepis S.F.Blake.

\section{Macrotomini}

Mallodon spinibarbis (Linnaeus, 1758)

Di Iorio \& Farina, 2009: 79

Hosts-FAGACEAE - Quercus palustris Münchh; LEGUMINOSAE - Albizia julibrissin Durazz., Gleditsia triacanthos L., Robinia pseudoacacia L.; PLATANACEAE - Platanus acerifolia (Aiton) Willd.; SALICACEAE - Populus alba L., P. nigra var. italica Koehne; SANTALACEAE - Jodina rhombifolia (Hook. \& Arn.) Reissek; SIMAROUBACEAE - Ailanthus altissima (Mill.) Swingle; ULMACEAE - Ulmus sp.

\section{Prionini}

Apterocaulus heterogama durnfordii

(Burmeister, 1879)

Barriga et al., 1993: 84; Di Iorio, 2004: 79 
Hosts-AMARANTHACEAE - Atriplex lampa (Moq.) Gillies ex D. Dietr.; APIACEAE - Mulinum spinosum Pers.; COMPOSITAE - Senecio sp.; LEGUMINOSAE - Anarthrophyllum rigidum (Hook. \& Arn.) Hieron.

\section{Apterocaulus heterogama heterogama (Burmeister, 1861)}

Di Iorio, 2004: 79

Host-SALICACEAE - Populus sp.

\section{VESPERIDAE}

\section{ANOPLODERMATINAE}

\section{Anoplodermatini}

\section{Migdolus fryanus Westwood, 1863}

Wilcken et al., 2005: 171.

Host-PINACEAE - Pinus caribaea var. hondurensis (Sénécl.) W. H.Barrett \& Golfari.

\section{Sypilus orbignyi Guérin-Méneville, 1840}

Duffy, 1960: 50; Di Iorio, 2004: 17.

Host-LEGUMINOSAE-Geoffroea decorticans (Hook. \& Arn.) Burkart.

\section{DISCUSSION}

In relation to Monné (2001a-c, 2002a-b, 2004), among all the species listed here, 96 were not previously cited and 115 new hosts are cited. Obriini (Cerambycinae) now has records for three species: Obrium multifarium Berg, 1889, Obrium trifasciatum Bosq, 1951 and Obrium vicinum Gounelle, 1909.

Regarding the host plants, a total of 259 species distributed among 188 genera and 69 families are listed. Leguminosae was the most representative family, with 67 species in 39 genera, followed by Malvaceae ( 12 species in 8 genera) and Sapotaceae (15 species in 7 genera). Species of economic importance are included on the list, such as avocado (Persea americana Mill.), mate (Ilex paraguariensis A. St.-Hil.), mango (Mangifera indica L.), jacaranda (Jacaranda sp.) and tamarind (Tamarindus indica L.).

Smodicum cucujiforme (Say, 1826) is considered an introduced species in Argentina and Uruguay, with its type locality in the United States of America. Its distribution extends from the Atlantic coast to the Mississippi Valley and Texas (Monné 2005).

\section{ACKNOWLEDGMENTS}

The authors are grateful to Miguel A. Monné for his help and support; to Michael Pratts for reviewing the abstract; to CNPq for the scholarships granted to the authors. Richard Boike revised the English text.

\section{REFERENCES}

Abreu, R.; B. Ronchi-Teles; M. A. Monné \& B. Vianez. 2009. First record of species of Cerambycidae (Coleoptera) in cardeiro (Scleronema micranthum) (Bombacaceae) in the Central Amazon. Neotropical Entomology 38: 432-433.
Barriga, J. E. \& D. Cepeda. 2005. Revisión del género Oectropsis Blanchard, 1851 (Coleoptera, Cerambycidae) con descripción de una nueva especie. Revista Chilena de Entomologia 31: 43-54.

Barriga, J. E.; T. Curcovic; T. Fichet; J. L. Henríquez \& J. Macaya. 1993. Nuevos antecedentes de coleópteros xilófagos y plantas hospederas en Chile, con una recopilación de citas prévias. Revista Chilena de Entomologia 20: 65-91.

Berkov, A. \& M. A. Monné. 2010. A new species of Neobaryssinus Monné \& Martins, and two new species of Baryssiniella new genus (Coleoptera: Cerambycidae), reared from trees in the Brazil nut family (Lecythidaceae). Zootaxa 2538: 47-59.

Bouchard, P.; Y. Bousquet; A. E. Davies; M. A. Alonso-Zarazaga; J. F. Lawrence; C. H. C. Lyal; A. F. Newton; C. A. M. Reid; M. Schmitt; A. Slipnski \& A. B. T. Smith. 2011. Famlily-group names in Coleoptera (Insecta). Zookeys 88: 1-972.

Casari, S. A. \& A. Santos-Silva. 2010. Larva of Setuceros aquilus (Thomson) (Coleoptera, Cerambycidae, Prioninae, Callipogonini). Neotropical Entomology 39: 746-751.

Casari, S. A. \& E. A. Steffanello. 2010. W. Immature of Tapuruia felisbertoi Lane, 1973 (Cerambycidae, Cerambycinae, Hexoplonini). Papéis Avulsos de Zoologia 50: 425-434.

Chalumeau, F. \& J. Touroult. 2005. Les Longicornes des Petites Antilles (Coleoptera, Cerambycidae) Taxonomie, éthologie, biogéographie. Pensoft, $241 \mathrm{p}$.

Clarke, R. O. S. \& S. Zamalloa. 2009. Life cycle of Phoebemima ensifera Tippmann (Coleoptera, Cerambycidae). Revista Brasileira de Entomologia 53: 287-290.

Dalens, P. H. 2011. Un nouveau Paranyssicus Martins, 2005 de Guyane (Coleoptera, Cerambycidae, Cerambycinae). L' Entomologiste 67: 2933.

Dalens, P. H.; G. L. Tavakilian \& J. Touroult. 2010. Révision des Compsocerini Thomson, 1864 de Guyane (Coleoptera,Cerambycidae,Cerambycinae). Les Cahiers Magellanes 2: 79-111.

Di Iorio, O. 1994. Cerambycidae (Coleoptera) y plantas hospedadoras del noroeste de Argentina. Revista Brasileira de Entomologia 38: 1522.

Di Iorio, O. 1997. Plantas hospedadoras y biogeografia de Cerambycidae (Coleoptera) del noroeste y centro de la Argentina. Revista de Biologia Tropical 44/45: 149-158.

Di Iorio, O. 2004. Cerambycidae, p. 17-79. In: H. A. Cordo, G. Logarzo, K. Braun \& O. Di Iorio. Catálogo de insectos fitófagos de la Argentina y sus plantas asociadas. Sociedad Entomológica Argentina, $734 \mathrm{p}$.

Di Iorio, O. 2006. New records, remarks and corrections to host plants of Cerambycidae (Coleoptera) from Argentina. Giornale Italiano di Entomologia 11: 159-178.

Di Iorio, O. \& J. Farina. 2009. Plantas hospedadoras de Cerambycidae (Coleoptera) de la provincia de Buenos Aires, Argentina. Revista del Museo Argentino de Ciencias Naturales 11: 77-99.

Duffy, E. A. J. 1960. A monograph of the immature stages of Neotropical timber beetles (Cerambycidae). British Museum (Natural History), vii $+327 \mathrm{p}$.

Fernandes, G. W. \& J. C. Santos. 2009. Feeding and mating behavior of Dorcacerus barbatus (Olivier, 1790) (Coleoptera: Cerambycidae) on Lantana camara L. (Verbenaceae). Lundiana 9: 81-84.

Fonseca, E.; S. Devesa \& A. Barro. 2008. Primer registro de Oxymerus aculeatus lebasi Dupont 1838 en Cuba (Coleoptera: Cerambycidae), con observaciones acerca de su historia natural. Boletín Sociedad Entomológica Aragonesa 43: 510-512.

Galileo, M. H. M. \& U. R. Martins. 2006. Inventário descritivo, p. 65-309. In: Galileo, M. H. M. \& U. R. Martins (org.). Cerambycidae (Coleoptera, Insecta) do Parque Copesul de Proteção Ambiental, Triunfo, Rio Grande do Sul, Brasil. Museu de Ciências Naturais da Fundação Zoobotânica do RS, 316 p.

Ganci, C. V. 2010. Oncideres germari Thomson (Cerambycidae-Coleoptera) nueva plaga urbana de Mendoza y San Juan. Multequina 19: 89-91.

Giacomel, F. 1979. Sobre a biologia de Compsosoma perpulchrum e Compsosoma phaleratum (Coleoptera, Cerambycidae). Dusenia 11: 95-101. 
González, O. E. \& O. Di Iorio, 1997. Plantas huespedes de Cerambycidae (Coleoptera) en el noroeste de Argentina. Revista de Biologia Tropical 44/45: $167-175$.

Logarzo, G.; D. Gandolfo \& H. Cordo. 2002. Biology of Apagomerella versicolor (Boheman) (Coleoptera, Cerambycidae) in Argentina, a candidate for biological control of cocklebur (Xanthium spp.). Biological Control 25: 22-29.

MacRae, T. C. \& M. E. Rice. 2007. Biological and distributional obervations on North American Cerambycidae (Coleoptera). Coleopterists Bulletin 61: 227-263.

Marinoni, R. C. 1969. Sobre alguns Cerambycidae (Coleoptera) e suas plantas hospedeiras. Ciência e Cultura 21: 470-471.

Martins, U. R. 1997. Cerambycidae Sul-Americanos (Coleoptera) Taxonomia.Vol. 1, Sociedade Brasileira de Entomologia, 217 p.

Martins, U. R. 2002. Tribo Achrysonini, p. 37-144. In: U. R. Martins (org.). Cerambycidae Sul-americanos (Coleoptera) Taxonomia. Vol. 4, Sociedade Brasileira de Entomologia, 265 p.

Martins, U. R. 2006. Tribo Hexoplonini, p. 21-211. In: U. R. Martins (org.). Cerambycidae Sul-Americanos (Coleoptera) Taxonomia. Vol. 8, Sociedade Brasileira de Entomologia, 234 p.

Mecke, R. 2002. Insetos do Pinheiro brasileiro-Insekten der brasilianischen Araukarie-Insects of the Brasilian Pine. Attempto Service GmbH, Tübingen, 79 p.

Monné, M. A. 2001a. Catalogue of the Neotropical Cerambycidae (Coleoptera) with known host plant - Part I: Subfamily Cerambycinae, Tribes Achrysonini to Elaphidiini. Publicações Avulsas do Museu Nacional 88: 1-108.

Monné, M. A. 2001b. Catalogue of the Neotropical Cerambycidae (Coleoptera) with known host plant - Part II: Subfamily Cerambycinae, Tribes Graciliiini to Trachyderini. Publicações Avulsas do Museu Nacional 90: 1-119.

Monné, M. A. 2001c. Catalogue of the Neotropical Cerambycidae (Coleoptera) with known host plant - Part III: Subfamily Lamiinae, Tribes Acanthocinini to Apomecynini. Publicações Avulsas do Museu Nacional 92: 1-94.

Monné, M. A. 2002a. Catalogue of the Neotropical Cerambycidae (Coleoptera) with known host plant - Part IV: Subfamily Lamiinae, Tribes Batocerini to Xenofreini. Publicações Avulsas do Museu Nacional 94: 1-92.

Monné, M. A. 2002b. Catalogue of the Neotropical Cerambycidae (Coleoptera) with known host plant - Part V: Subfamilies Prioninae, Parandrinae, Oxypeltinae, Anoplodermatinae, Aseminae and Lepturinae. Publicações Avulsas do Museu Nacional 96: 1-70.

Monné, M. A. 2004. Catalogue of the Neotropical Cerambycidae (Coleoptera) with known host plant - Part VI: Host plant index. Publicações Avulsas do Museu Nacional 100: 1-95.

Monné, M. A. 2005. Catalogue of the Cerambycidae (Coleoptera) of the Neotropical Region. Part I. Subfamily Cerambycinae. Zootaxa 946: $1-765$.

Monné, M. A.; M. Bianchi; A. Sánchez; R. Escudero. 2002. Cerambícidos (Coleoptera) que atacan Eucalyptus globulus y Eucalyptus grandis en Uruguay. Agrociencia 6: 63-68.

Monné, M. A. \& M. L. Monné. 2006. Novos táxons sul-americanos de Parmenini e Pteropliini (Coleoptera, Cerambycidae, Lamiinae). Revista Brasileira de Entomologia 50: 488-491.

Monné, M. A. \& M. L. Monné. 2010. Synopsis of the genus Colobothea Lepeletier \& Audinet-Serville in Bolivia, with descriptions of two new species and new country records. Zootaxa 2542: 33-47.

Monné, M. L.; M. A. Monné \& J. R. M. Mermudes. 2009. Inventário das espécies de Cerambycinae (Insecta. Coleoptera, Cerambycidae) do Parque Nacional do Itatiaia, RJ, Brasil. Biota Neotropica 9: 1-30.

Morris, R. F. 2002. Distribution and biological notes for some Cerambycidae (Coleoptera) occurring in the southeastern United States. Insecta Mundi 16: 209-213

Napp, D. S. 1994. Phylogenetic relationships among the subfamilies of Cerambycidae (Coleoptera, Chrysomeloidea). Revista Brasileira de Entomologia 38: 265-419.
Néouze, G. L. \& G. L. Tavakilian. 2005. Matériaux pour une révision des Xenofreini - I. Espéces nouvelles de Guyane (Lamiinae). Coléoptères 11: $129-164$

Néouze, G. L. \& G. L. Tavakilian. 2010. Nouvelles espèces du genre Oreodera Audinet-Serville, 1835 de Guyane française (Coleoptera, Cerambycidae, Lamiinae). Coléoptères 16: 43-74.

Olckers, T; J. C. Medal \& D. E. Gandolfo. 2002. Insect herbivores associated with species of Solanum (Solanaceae) in northeastern Argentina and southeastern Paraguay, with reference to biological control of weeds in South Africa and the United States of America. Florida Entomologist 85: 254-260.

Paulino Neto, H. F.; G. Q. Romero \& J. Vasconcellos Neto. 2005. Interactions between Oncideres humeralis Thomson (Coleoptera: Cerambycidae) and Melastomataceae: Host-Plant selection and patterns of host use in South-East Brazil. Neotropical Entomology 34: 7-14.

Peñaherrera-Leiva, A. Y. \& G. L. Tavakilian. 2003. Nouvelles espèces et nouveaux genres de Rhinotragini (Coleoptera, Cerambycidae). I. Coléoptères 9: 163-214

Peñaherrera-Leiva, A. Y. \& G. L. Tavakilian. 2004. Nouvelles espèces et nouveaux genres de Rhinotragini (Coleoptera, Cerambycidae). III. Coléoptères 10: 119-150.

Rizzuto, S. 2009. Observations on the larval biology in the genus Calydon (Coleoptera: Cerambycidae) with new records in Patagonia, Argentina. Revista de la Sociedad Entomológica Argentina 68: 391-396.

Roddick, C.; N. Rodriguez \& A. Berkov. 2009. Hard-won host record for two species of Trypanidius Blanchard, 1843 (Coleoptera, Cerambycidae). Coleopterists Bulletin 63: 105-107.

Silva, A. G.; C. R. Gonçalves; D. M. Galvão; A. J. L. Gonçalves; J. Gomes; M. N. Silva \& L. Simoni, 1968. Quarto catálogo dos insetos que vivem nas plantas do Brasil. Seus parasitos e predadores. Rio de Janeiro, Ministerio de Agricultura, 622 p.

Souza, M. F.; D. Gabriel \& J. A. Azevedo. 2001. Caracterização morfológica de três espécies de broca-da-haste em chuchuzeiro (Coleoptera, Cerambycidae, Lamiinae). Neotropical Entomology 30: 475-477.

Svácha, P.; J. Wang \& S. Chen. 1997. Larval morphology and biology of Philus antenatus and Heterophilus punctulatus, and systematic position of the Philinae (Coleoptera: Cerambycidae and Vesperidae). Annales de la Société Entomologique de France 33: 323-369.

Tavakilian, G. L.; A. Berkov; B. Meurer-Grimes \& S. Mori. 1997. Neotropical tree species and their faunas of xylophagous longicorns (Coleoptera: Cerambycidae) in French Guiana. Botanical Review 63: 304-355.

Tavakilian, G. L.; P. H. Dalens \& J. Touroult. 2007. Les Rhaphiptera AudinetServille, 1835, en Guyane et description d'un nouveau genre affine (Coleoptera, Cerambycidae, Lamiinae, Pteropliini). Les Cahiers Magellanes 72:1-19.

Tavakilian, G. L. \& G. L. Néouze. 2004. Les Desmiphora Audinet-Serville, 1835, de Guyane; descriptions d'espèces nouvelles (Coleoptera, Cerambycidae, Lamiinae). Coléoptères 10: 49-68.

Tavakilian, G. L. \& G. L. Néouze. 2011. Le genre Oreodera AudinetServille, 1835, en Guyane (Coleoptera, Cerambycidae, Lamiinae, Acanthoderini). Les Cahiers Magellanes 3: 52-107.

Tavakilian, G. L. \& A. Y. Peñaherrera-Leiva. 2003a. Nouvelles espèces et nouveaux genres de Rhinotragini (Coleoptera, Cerambycidae). II. Coléoptères 9: 275-314.

Tavakilian, G. L. \& A. Y. Peñaherrera-Leiva. 2003b. Révision du genre Pycnomorphus Thomson, 1864. Systématique et phylogénie (Lamiinae, Acanthoderini). Annales de la Société Entomologique de France 39: 3-24.

Tavakilian, G. L. \& A. Y. Peñaherrera-Leiva. 2005. Nouvelles espèces et nouveaux genres de Rhinotragini (Coleoptera, Cerambycidae). IV. Coléoptères 11: 27-50.

Tavakilian, G. L. \& A. Y. Peñaherrera-Leiva. 2007. Nouvelles espèces et nouveaux genres de Rhinotragini (Coleoptera, Cerambycidae). V. Coléoptères 13: 79-122.

The Plant List. 2010. Version 1. Published on the Internet. Available at: http://www.theplantlist.org/ (acessed 25 April 2011). 
Touroult, J. 2004. Les longicornes associés aux rameaux coupés par Oncideres amputator en Guadeloupe (Coleoptera, Cerambycidae). Le Coléoptèriste 7: 129-134.

Villacide, J. M.; P. Sackmann \& M. Elgueta. 2006. Nuevo hospedador de Huequenia livida (Coleoptera: Cerambycidae) en Argentina. Revista de la Sociedad Entomológica Argentina 65: 122-125.

Vitali, F.; K. W. Wolf \& J. Haxaire. 2006. Biological and faunitic notes on the Jamaican population of Steirastoma histrionicum White, 1855 (Coleoptera, Cerambycidae). Lambillionea 106: 661-667.

Wilcken, C. F.; C. Orlato \& A. L. T. Ottati. 2005. Ocorrência de Migdolus fryanus (Coleoptera: Cerambycidae) em plantios de Pinus caribaea var. hondurensis. Revista Árvore 29: 171-173.

Zanuncio, J. C.; R. P. Almada; M. F. Sossai; R. Zanetti; T. V. Zanuncio \& M. C. Fialho. 2005. Sphallenum tuberosum (Coleoptera, Cerambycidae) em plantas de Eucalyptus spp. no Município de Prado, Bahia. Revista Árvore 29: 339-343.

Zanuncio, J. C.; M. E. Pires; R. P. Almado; R. Zanetti; M. A. Monné; J. M. M. Pereira \& J. E. Serrão. 2009. Damage assessment and host plant records of Oxymerus basalis (Dalman, 1823) (Cerambycidae: Cerambycinae: Trachyderini) in Brazil. Coleopterists Bulletin 63: 179-181. 